\title{
Iron Oxide and Gold Nanoparticles in Cancer Therapy
}

\author{
Irena Gotman ${ }^{1,2, a)}$, Sergey G. Psakhie ${ }^{2,3}$, Aleksandr S. Lozhkomoev², \\ and Elazar Y. Gutmanas ${ }^{1,2, b)}$ \\ ${ }^{I}$ Department of Materials Science and Engineering, Technion-Israel Institute of Technology, Haifa, 32000 Israel \\ ${ }^{2}$ Tomsk Polytechnic University, Tomsk, 634050 Russia \\ ${ }^{3}$ Institute of Strength Physics and Materials Science SB RAS, Tomsk, 634055 Russia \\ a) Corresponding author: gotman@technion.ac.il \\ b) gutmanas@technion.ac.il
}

\begin{abstract}
Continuous research activities in the field of nanomedicine in the past decade have, to a great extent, been focused on nanoparticle technologies for cancer therapy. Gold and iron oxide nanoparticles (NP) are two of the most studied inorganic nanomaterials due to their unique optical and magnetic properties. Both types of NPs are emerging as promising systems for anti-tumor drug delivery and for nanoparticle-mediated thermal therapy of cancer. In thermal therapy, localized heating inside tumors or in proximity of tumor cells can be induced, for example, with Au NPs by radiofrequency ablation heating or conversion of photon energy (photothermal therapy) and in iron oxide magnetic NPs by heat generation through relaxation in an alternating magnetic field (magnetic hyperthermia). Furthermore, the superparamagnetic properties of iron oxide nanoparticles have led to their use as potent MRI (magnetic resonance imaging) contrast agents. Surface modification/coating can produce NPs with tailored and desired properties, such as enhanced blood circulation time, stability, biocompatibility and water solubility. To target nanoparticles to specific tumor cells, NPs should be conjugated with targeting moieties on the surface which bind to receptors or other molecular structures on the cell surface. The article presents several approaches to enhancing the specificity of Au and iron oxide nanoparticles for tumor tissue by appropriate surface modification/functionalization, as well as the effect of these treatments on the saturation magnetization value of iron oxide NPs. The use of other nanoparticles and nanostructures in cancer treatment is also briefly reviewed.
\end{abstract}

\section{INTRODUCTION}

The multidisciplinary field of Nanotechnology involving physics, chemistry, biology and engineering, has opened new horizons in diagnostics and therapeutics, and especially in the diagnosis and treatment of cancer [1-6]. After cardiovascular diseases, cancer is the second leading cause of death worldwide. Despite rapid developments of diagnostic techniques, surgery techniques, pharmaceutical chemistry and chemotherapy, detection and therapy of cancer at the initial stage remains a major challenge. In the last decade, great attention has been focused on development of nanoparticles and nanoparticle-targeting molecules systems for drug delivery [1-14], hyperthermia tumor treatment [15-19] and MRI-magnetic resonance imaging diagnostics [20-24]. New approaches were successfully introduces in cancer therapy.

Compared to micron-scale particles, nanoparticles are better suited for intravenous administration. They are much smaller than the smallest capillaries $(5 \mu \mathrm{m})$ and thus can pass through capillary systems of organs and tissues without the risk of vessel embolism. Nanoparticles (diameter $<100 \mathrm{~nm}$ ) are generally less recognized by the immune system and can circulate for several hours in the blood stream. Due to their small size, nanoparticles are able to diffuse through fenestrations of the altered blood vessels found in diseased tissues (Fig. 1), cross biological membranes and access cells, tissues and organs that micron-sized particles normally cannot. Small nanoparticles $(10-100 \mathrm{~nm})$ that avoid recognition ('stealth nanoparticles') can accumulate in neoplastic tissues-'enhanced permeability and retention' (EPR) effect. Particles $\geq 100 \mathrm{~nm}$ are rapidly removed from circulation by mononuclear phagocytic cells, located primarily in the liver and spleen. Such particles accumulate in liver and spleen before they are able to reach the site of disease (hepatic filtration) [25]. Particles $<5.5 \mathrm{~nm}$ are cleared by excretion (renal filtration-kidneys) [26].

Physics of Cancer: Interdisciplinary Problems and Clinical Applications (PC'16) AIP Conf. Proc. 1760, 020020-1-020020-6; doi: 10.1063/1.4960239

Published by AIP Publishing. 978-0-7354-1418-1/\$30.00 


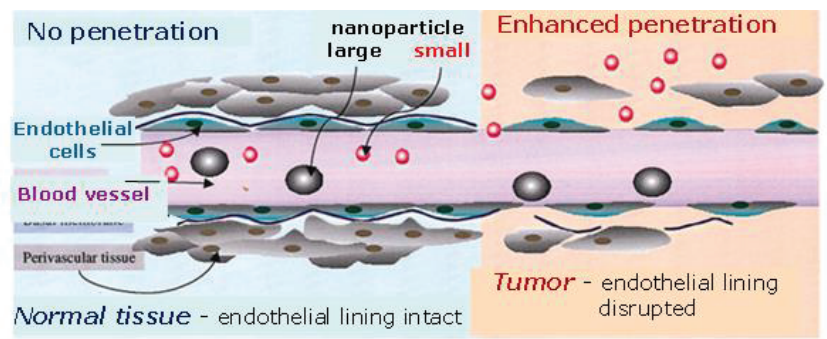

FIGURE 1. A schematic of enhanced permeability and retention effect (EPR)-

passive extravasation of nanoparticles through leaky tumor vasculature

Currently there are three major applications of nanoparticles in cancer therapy:

(1) drug delivery;

(2) magnetic and radio-frequency (RF) hyperthermia of cancer;

(3) magnetic resonance imaging (MRI).

Gold and iron oxide nanoparticles (NP) are the two most studied inorganic nanomaterials. Both are biocompatible, relatively non-toxic, possess unique physical properties and are emerging as promising systems for antitumor drug delivery and for nanoparticle-mediated thermal therapy of cancer. Iron oxide NPs are also used in magnetic resonance imaging (MRI) of tumors. It can be mentioned that as a result of metabolic degradation of iron oxide NPs iron ions are added to the body's iron stores and eventually incorporated by erythrocytes as hemoglobin allowing for their safe use in vivo.

In addition to gold and iron oxide NPs, mesoporous silica [14, 27], carbon nanotubes [28, 29], CdS, CdSe nanoparticles [30] as well as natural therapeutics such as Nisin [31] and Curcumin [32, 33] are also studied in antitumor treatments. Hollow nanoparticles are used for encapsulation of antitumor drugs [14, 34]. To target nanoparticles to specific tumor cells, NPs are conjugated with targeting moieties on the surface that bind to receptors or other molecular structures on the cell's surface [1-12]. In many cases, combination of NPs drug delivery with magnetic resonance imaging (MRI), MRI with magnetic hyperthermia as well as photothermal and radiofrequency ablation therapy with chemotherapy are employed in cancer therapy.

\section{TARGETED NANOPARTICLES-DRUG DELIVERY TO TUMORS}

For their effective use in medical applications in general and in cancer therapy and diagnostics in particular, nanoparticles should be preferably bio-functionalized. In the last decade, surface functionalization of nanoparticles with biomolecules for targeted drug delivery to tumors has been a top priority in biomedical technology. Targeted delivery of nanoparticles to a specific tissue can be achieved by conjugation with particular biological ligands that will direct the particle in the bloodstream to the tumor (see schematic in Fig. 2). Also shown in the figure is the transformation of the targeted NP into a drug-delivery vehicle by attaching an anti-cancer drug to the particle surface.

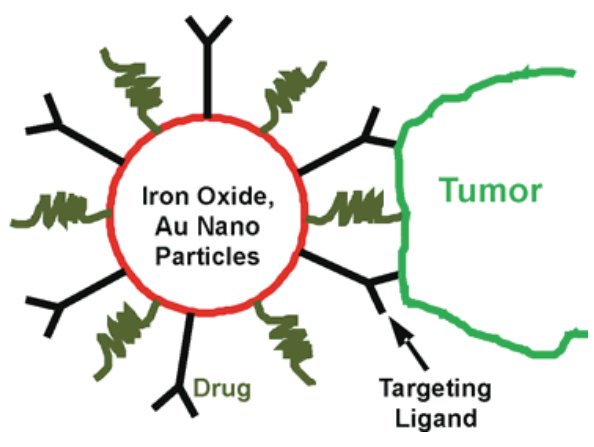

FIGURE 2. A schematic of NP surface bio-functionalization for active tumor targeting

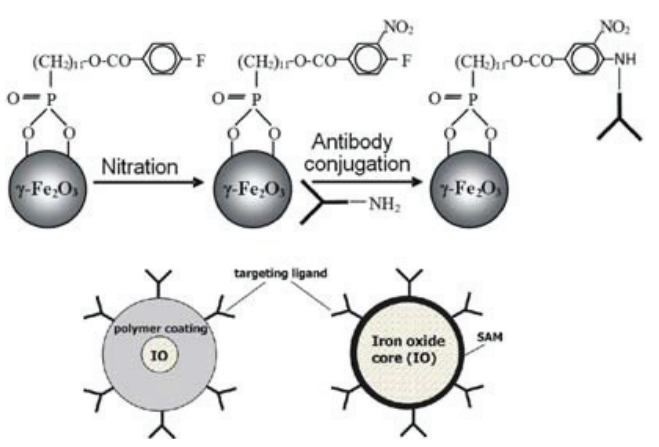

FIGURE 3. A schematic of iron oxide NP biofunctionalization for targeting toward diseased cells: attachment of a Phosphonate-Anchored SAM having an antibody binding tail [37] 


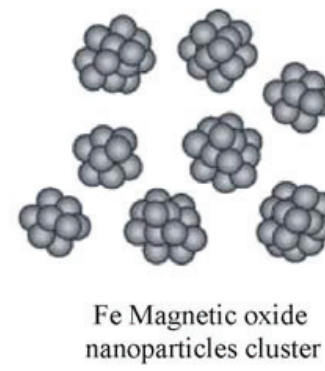

(a)

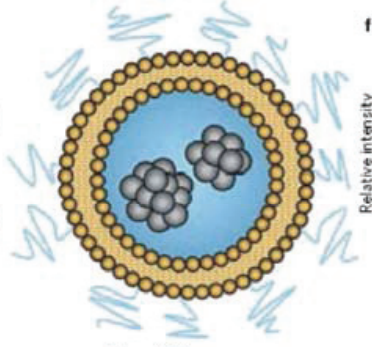

Ferri-liposome

(b)

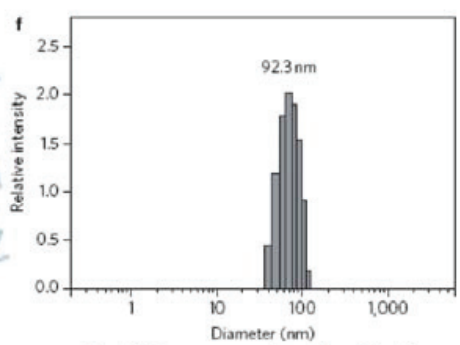

Ferri-liposome size distribution Lipid layers about $10 \mathrm{~nm}$

(c)

FIGURE 4. Schematics of (a) magnetic iron oxide NP's clusters, (b) ferri-liposome and (c) ferri-liposome size distribution [40]

Nanoparticles allow for in vivo delivery of challenging drugs (nucleic acids, peptides, proteins, molecules difficult to solubilize). The therapeutic indices of drugs can be increased after association with nanoparticles, and the bio-availability and stability of drugs can be improved in nanoparticles. Targeted nanoparticles can deliver drugs directly to diseased organs and into drug-resistant cells. In a targeted NP, the drug is encapsulated inside the core to protect the drug from being degraded in the body before it reaches its target and to prevent the drug from interacting with normal cells, thus avoiding side effects. The surface characteristics of a NP shell should allow the attachment of molecules that bind particular cellular receptors for the nanoparticle to actively target cells expressing the receptor.

Iron oxide nanoparticles (IONPs) consisting of maghemite $\left(\gamma-\mathrm{Fe}_{2} \mathrm{O}_{3}\right)$ and/or magnetite $\left(\mathrm{Fe}_{3} \mathrm{O}_{4}\right)$ have been extensively studied due to their low toxicity and a unique magnetic property - superparamagnetism. Single domain IONPs become superparamagnetic below a critical size of 30-50 nm: they may have high saturation magnetization and, as a result, high magnetic energy when an external magnetic field is applied, and at the same time practically zero residual magnetization. Successful in vivo usage of IONPs requires particles below $100 \mathrm{~nm}$ in size that are well dispersed in physiological media.

Since bare IONPs are not stable in water or in physiological fluids, they tend to agglomerate and precipitate quickly [35]. To promote dispersion in aqueous media, iron oxide cores are often coated with polymers, for example, dextran [36]. These organic layers can also provide NP's with functional terminal groups that can be used for attaching targeting agents. One drawback of the surface engineered IONPs is that the polymer coatings cause a significant increase in the overall hydrodynamic diameter of nanoparticles, resulting in decreased magnetic contrast. In [37] it was shown that application of phosphonate-anchored ultrathin self-assembled monolayers (SAMs) as coatings for such particles results in increase of superparamagnetic core diameter with overall diameter being within the required nanoscale range (Fig. 3). This should lead to a substantial increase of magnetic energy and increase of magnetic contrast. Magnetic nanoparticles encapsulated within a phospholipid bilayer, forming liposomes, have been reported to have considerable structural and pharmacokinetic advantages for drug delivery [38, 39]. Owing to their ability to encapsulate both hydrophobic and hydrophilic therapeutics, liposomes prevent local dilution of the drug and limit its interaction with the surrounding environment, enabling reduction of the therapeutic dose and toxicity. An example of using iron oxide NP clusters encapsulated inside liposomes was reported in [40] (Fig. 4). Under the influence of an external magnet, such ferri-liposomes target both the tumor and its microenvironment, substantially reducing the size of the tumor in a mouse after treatment.

Multi-functional gold $\mathrm{(Au}$ ) NPs are highly stable and versatile scaffolds for drug delivery due to their unique size, chemical and physical properties. Surface bio-functionalization of Au nanoparticles provides the possibility of targeting specific cells and thus destructing cancer cells (Fig. 2). Gold NPs have advantages in binding targeting ligands. A number of different types of ligands including branched molecules, dendrimers, can be attached to Au nanoparticles, in order to target different tumor cells. The release of drugs from gold NPs can be controlled by external light in the wave length range where tissues are transparent (near-infrared, NIR), or by intracellular glutathione mediate release [41-43] (Fig. 5) (glutathione is an important antioxidant preventing damage to some important cellular components). The presence of certain hormone receptors on cancer cells can be utilized for active targeting of nanoparticles. In [44], for example, the delivery efficiency of an anti-tumor drug was significantly improved through decorating $\mathrm{Au}$ NPs with specific ligands that can recognize and selectively bind to estrogen receptors overexpressed in breast cancer cells and to androgen receptors over expressed in prostate cancer cells. 


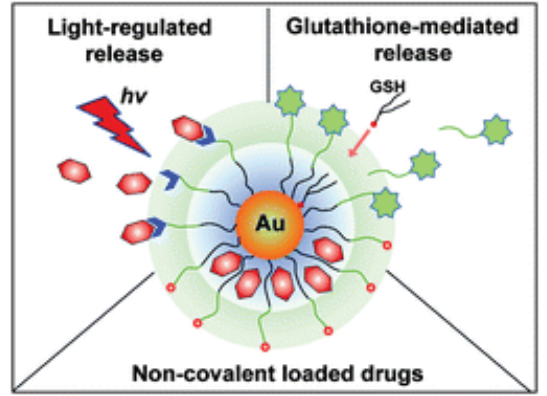

FIGURE 5. Schematic mechanisms of drug release from $\mathrm{Au}$ NPs [41]

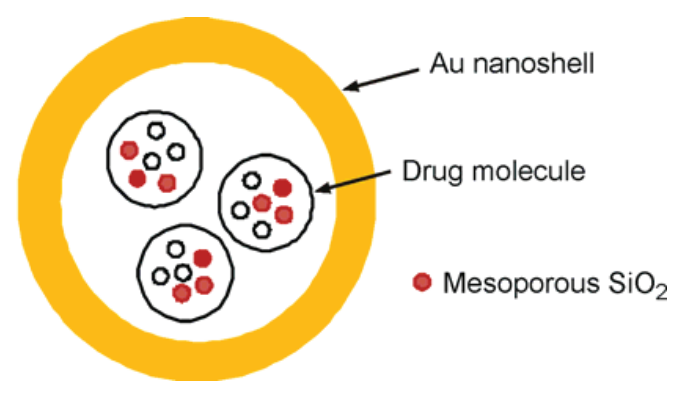

FIGURE 6. A schematic of rattled mesoporous silica NP in gold nanoshell for targeted drug release

Mesoporous silica NPs and rattled mesoporous silica NPs in gold nanoshells (see schematic in Fig. 6) are also used in drug delivery and chemotherapy of cancer [14, 27, 44].

\section{NANOPARTICLES-HYPERTHERMIA TREATMENT OF CANCER}

\section{Magnetic Hyperthermia}

Superparamagnetic NPs, such as iron oxide NPs, or magnetic nanoparticles, such as, for example, cobalt ferrite $\mathrm{CoFe}_{2} \mathrm{O}_{4}$, NPs [45], can generate heat through relaxation in an alternating magnetic field. The heating effects are related to losses during the magnetization reversal process and/or to hysteresis losses. Magnetic hyperthermia is a promising treatment of tumors. As discussed above (see Figs. 2 and 3), targeting ligands can direct iron oxide or magnetic NPs to the tumor. Upon application of an external AC magnetic field, the heat generated by magnetic nanoparticles is transferred into the neighboring diseased tissue, and, if the temperature is locally maintained above $42^{\circ} \mathrm{C}$ for more than half an hour, the tumor cells are destroyed or, at least, their growth is reduced [46]. The heat generated in the vicinity of superparamagnetic iron oxide or magnetic nanoparticles by induced eddy current is very small and should not damage normal cells [47]. To burn away the cancer cells, the frequency of alternating magnetic field and exposure should be adjusted for each type of nanoparticle. A schematic of application of magnetic nanoparticles for Hyperthermia therapy and MRI diagnosis of tumors is shown in Fig. 7.

\section{Radio-Frequency (RF) Heating Hyperthermia-RF Ablation}

Selective heating of conducting but non-magnetic nanoparticles can be achieved by application of a radiofrequency alternating electric field resulting in eddy currents. The phenomenon is known as induction heating or high frequency induction heating and is used in processing metal alloy parts as well as parts made of other conducting materials such as carbon and carbon-fiber composites.

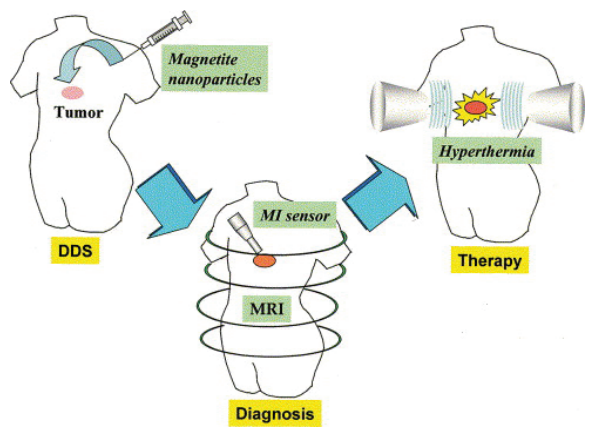

FIGURE 7. A schematic of magnetic nanoparticles application for Hyperthermia therapy and MRI diagnosis of tumors

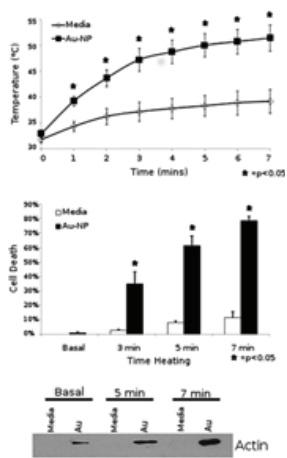

FIGURE 8. In vitro liver cancer HepG2 cells exposed to $35 \mathrm{~W}$ in the RF field in the presence or absence of Au NPs in water solution as a function of time [48] 
The eddy current and the generated heat depend on electric resistance. Since the resistance of nanoparticles is high, the currents are very small. This may be advantageous when only small overheating is needed to destroy cancer cells without damaging normal cells. Damage and destruction of cancer or other disfunctional tissues by applying a radio-frequency electromagnetic field is called RF ablation. Radiofrequency heating of gold NPs is widely used in research and clinical practice for cancer therapy [4, 12, 13, 48, 49]. For example, 80\% of HepG2 liver cancer cells cultured in solutions containing gold NPs heated by $35 \mathrm{~W}$ RF died upon a 7 min exposure vs. only $15 \%$ of cells in the control water solution [48] (see Fig. 8). Due to the unique property of localized surface plasmon resonance, gold nanoparticles (including gold nanoshells and nanorods) produce heat when excited by visible-NIR light. This photothermal energy conversion makes Au NPs excellent probes for hyperthermia application in cancer treatment [49]. Carbon nanotubes absorb infrared radiation (IR) in the range between 700 and $1100 \mathrm{~nm}$, where body tissues are most transparent. Absorbed IR promotes molecular oscillation leading to local heating of the surrounding tissue-hyperthermia, and destruction of cancer cells [50].

\section{IRON OXIDE NANOPARTICLES-MRI CONTRAST AGENTS}

Magnetic resonance imaging (MRI) is a non-invasive diagnostic medical imaging technique (schematic in Fig. 7) that uses the interaction between strong magnetic field RF pulses and hydrogen nuclei in the body tissues. When a strong magnetic field is applied, hydrogen nuclei align with the direction of this field and create a net magnetic moment. Once the RF signal is removed, the hydrogen nuclei realign themselves with the magnetic field in the process called relaxation. When the hydrogen nuclei relax and lose their energy, they emit a radio wave signal which can be detected by an external coil and then converted to an image in the MRI system. MRI imaging sensitivity can be greatly improved via administration of contrast agents. Over the last decade, much attention has been devoted to the development of superparamagnetic iron oxide (SPIO) NPs as contrast agents for MRI [6, 9, 10, 16, 24, 51]. Experiments are also going on with superparamagnetic Gd as a contrast agent [24]. In [52] Gd was deposited on single wall carbon nanotubes for MRI and hyperthermia therapy.

\section{CONCLUSION}

NPs are well suited for intravenous administration; they are able to diffuse through fenestrations of the altered blood vessels found in diseased tissues, cross biological membranes and access cells, tissues and organs that micronsized particles normally cannot.

Iron oxide and gold nanoparticles (NPs) are emerging as promising systems for anti-tumor drug delivery and for nanoparticle-mediated thermal therapy and diagnostics of cancer:

- targeted drug delivery;

- hypothermia-destruction of cancer cells by overheating;

- MRI-magnetic resonance imaging.

Active targeting of NPs by conjugation with specific ligands which have high affinity to receptors over expressed on cancer cells may facilitate their delivery to cancerous tumors and cells; drugs can be encapsulated in meso- or nanopores of NPs or attached to the NPs' surfaces.

Other NPs, such as mesoporous silica, carbon nanotubes, CdS/CdSe, polymers, as well as natural therapeutics, such as Nisin and Curcumin, are also studied as anticancer agents.

\section{ACKNOWLEDGMENTS}

The current paper is a mini-review paper: no experiments, including those on animals or humans were performed.

All individual participants discussed in this study, or for whom any identifying information or image has been presented, have freely given their informed written consent for such information and/or image to be included in the published article.

\section{REFERENCES}

1. R. Langer, Nature 392, 5-10 (1998).

2. O. C. Farokhzad and J. M. R. Langer, Adv. Drug Deliv. 58, 1456-1459 (2006).

3. R. Petros and J. DeSimone, Nature Rev. 9, 615-627 (2010). 
4. G. Bao, S. Mitragotri, and S. Tong, Ann. Rev. Biomed. Eng. 15, 253-282 (2013).

5. K-T. Yong, I. Roy, M. T. Swihart, and P. N. Prasad, J. Mater. Chem. 19, 4655-4672 (2009).

6. I. Berger, C. Dubernet, and P. Courveur, Adv. Drug Del. Rev. 64, 24-36 (2012).

7. L. Brannon-Peppas and J. O. Blanchette, Adv. Drug Del. Rev. 64, 206-212 (2012).

8. F. X. Gu, R. Karnik, A. Z. Wang, F. Alexis, E. Levy-Nissenbaum, et al., Nano Today 2(3), 14-21 (2007).

9. A. K. Gupta and M. Gupta, Biomaterials 26, 3995-4021 (2005).

10. M. Mahmoudi, S. Sant, B. Wang, S. Laurent, and T. Sen, Adv. Drug Del. Rev. 63, 24-46 (2011).

11. M. Arrueboa, R. Fernandez-Pachecoa, M. R. Ibarraa, and S. Santamaria, Nano Today 2(3), 22-32 (2007).

12. P. K. Jain, I. H. El-Sayed, and M. A. El-Sayed, Nano Today 2(3),18-29 (2007).

13. J. Cardinal, J. R. Klune, E. Chory, G. Jejabalan, J. S. Kanzius, and M. Nalesnik, Surgery 144, 125-132 (2008).

14. J. Lu, Z. Li, J. I. Zink, and F. Tamaori, Nanomedicine 8, 213-220 (2012).

15. J. Verma, S. Lal, and C. J. F. Van Noorden, Int. J. Nanomed. 9, 2863-2877 (2014).

16. R. Ghosh, L. Pradahan, Y. P. Devi, S. S. Meena, R. Tewari, et al., J. Mater. Chem. 21, 13388-13398 (2012).

17. C. Lou and D. Xing, Int. J. Hyperthermia 26(4), 338-346 (2010).

18. R. S. McCoy, S. Choi, G. Collins, B. J. Ackerson, and C. J. Ackerson, ACS Nano 7, 2610-2616 (2013).

19. S. Maluta, M. Romano, S. Dall'oglio, et al., Int. J. Hyperthermia 26(2), 108-117 (2010).

20. A. Gupta, R. S. Kane, and D-A. Borca-Tasciuc, J. Appl. Phys. 108, 064901 (2010).

21. S. Mornet, S. Vasseur, F. Grasset; P. Veverka, G. Goglio, et al., Prog. Solid State Chem. 34, $237-247$ (2006).

22. C. Sun., J. C. H. Lee, and M. Zhang, M. Adv. Drug Del. Rev. 60, 1252-1265 (2008).

23. C. Corot, P. Robert, J. M. Idee, and M. Port, Adv. Drug Del. Rev. 58, 1471-1504 (2006).

24. L. Li, R. Tong, M. Li, and D. S. Kohane, Acta Biomater. 33, 34-39 (2016).

25. M. Yokoyama, J. Artif. Organs. 8, 77-84 (2005).

26. M. Longmire, P. L. Choyke, and H. Kobayashi, Nanomed. 3, $703-717$ (2008).

27. M. Gary-Bobo, O. Hocine, D. Brevet, M. Maynadier, L. Raehm, et al., Int. J. Parm. 423, 509-515 (2012).

28. V. Kumar, G. Toffoli, and F. Rizzolio, ACS Med. Chem. Lett. 4, 1012-1013 (2013).

29. Z. Liu, J. T. Robinson, S. M. Tabakman, K. Yang, and H. Dai, Mater. Today 14, 316-323 (2011).

30. K-Y. Yong, Y. Wang, I. Roy, H. Rui, M. T. Swihart, et al., Theranostics 2, 681-694 (2012).

31. N. E. Joo, K. Ritchie, P. Kamarajan, D. Miao, and Y. L. Kapila, Cancer Medicine 1, 295-305 (2012).

32. R. Wilken, M. S. Veena, M. B. Wang, and E. S. Srivastan, Molecular Cancer 10, 2-19 (2011).

33. R. K. Maheshwari, A. K. Singh, J. Gaddipati, and R. C. Srimall, Life Sci. 78, 2081-2087 (2006).

34. G.-F. Luo, W.-H. Chen, Y. Liu, Q. Lei, R.-X. Zhuo, and X.-Z. Zhang, Sci. Rep. 4, 6064 (2014), doi 101038.

35. C. Boyer, M. R. Whittaker, V. Bulmus, V. J. Liu, and T. P. Davis, NPG Asia Mater. 2, 23-30 (2010).

36. M. Di Marco, C. Sadun, M. Port, I. Guilbert, P. Couvreur, and C. Dubernet, Int. J. Nanomed. 2, 609-622 (2007).

37. H. Benbenishty-Shamir, R. Tzur, I. Gotman, E. Y. Gutmanas, and C. Sukenik, Langmuir 27, 12082-12089 (2011).

38. J. W. Bulte, M. de Cuyper, D. Despres, and J. A. Frank, J. Magn. Reson. Imaging 9, 329-335 (1999).

39. D. Di Paolo, M. Loi, F. Pastorino, C. Brignole, D. Marimpietri, P. Becherini, et al., Methods Enzymol. 465, 225-249 (2009).

40. G. Mikhailov, U. Mikac, A. Magaeva, V. I. Itin, E. P. Naiden, I. Psakhye, L. Babes, T. Reinheckel, C. Peters, R. Zeiger, M. Bogio, V. Turk, S. G. Psakhye, B. Turk, and O. Vasiljeva, Nature Nanotech. 6, 594-602 (2011).

41. C.-K. Kim, P. Ghosh, and V. M. Rotello, Nanoscale 1, 61-67 (2009).

42. H. Liu, D. Chen, L. Li, T. Liu, L. Tan, X. Wu, and F. Tang, Angew. Chem. Int. Ed. 50, 891-895 (2011).

43. B. Singhana, P. Slattery, A. Chen, W. Michael, and M. P. Melancon, AAPS Pharm. Sci. Tech. 15, 741-752 (2014).

44. E. C. Dreaden, L.A. Austin, M. A. Mackey, and M. A. El-Sayed, Ther. Deliv. 3, 457-478 (2012).

45. D.-H. Kim, D. E. Nikles, D. T. Johnson, and C. S. Brazel, J. Magn. Magnetic Mater. 320, 2390-2396 (2008).

46. Y. Rabin, Int. J. Hyperthermia 18(3), 194 (2002).

47. S. Masashige, J. Biosci. Bioeng. 94, 606-613 (2002).

48. C. B. Collins, R. S. McCoy, B. J. Ackerson, G. J. Collins, and C. J. Ackerson, Nanoscale 6, 8459-8472 (2014).

49. L. R. Hirsch, R. J. Stafford, J. A. Bankson, S. R. Sershen, R. Rivera, et al., Pros. Natl. Acad. Sci. USA 100, 13549-13554 (2003).

50. N. H. Levi-Polyachenko, E. J. Merkel, B. T. Jones, D. I. Carrol, and J. H. Stewart IV, Mol. Pharm. 6, 10921099 (2009).

51. A. J. Cole, V. C. Yang, and A. E. David, Trend Biotech. 29, 323-332 (2011).

52. T. Peci, J. S. Dennis, and M. Baxendale, Carbon 87, 226-232 (2015). 